\title{
Lesões pré-ulcerativas gastroesofágicas em miniporcos Gastroesophageal ulcer research in mini pigs
}

\author{
Renato Luiz Silveira, ${ }^{* *}$ Ana Claudia de Menezes Cruz, ${ }^{* * *}$ Raphael Mansur Medina, ${ }^{* * * *}$ Jurandyr de Abreu Câmara Filho, ${ }^{* *}$ \\ Edmundo Jorge Abílio, ${ }^{* * * *}$ Eulógio Carlos Queiroz de Carvalho****
}

\section{Resumo}

Aúlcera gastroesofágica figura como uma das principais causas de morte súbita de suínos em fase de reprodução. O objetivo deste trabalho foi avaliar os achados anatomopatológicos das lesões gástricas subclínicas em miniporcos. Quarenta animais, com peso médio de $39 \mathrm{~kg}$ e idade de 17 meses, foram mantidos em biotério, em condições de temperatura e umidade constantes, alimentados duas vezes ao dia, sendo depois eutanasiados e necropsiados. Os estômagos foram colhidos e avaliados. Macroscopicamente, 29 animais apresentaram alterações na região aglandular, sendo que $37,5 \%$ foram classificadas no grau $1 ; 22,5 \%$, no grau 2 ; e 12,5\%, no grau 3. Microscopicamente, 29 (72,5\%) miniporcos apresentaram paraqueratose. Destes, 35\% tinham a forma moderada, $25 \%$ a discreta e $12,5 \%$ a acentuada. As lesões glandulares foram mais extensas na região cárdica, seguida da antral e fúndica. Trinta e seis (90\%) animais apresentaram lesão em pelo menos uma das três regiões. Em $21(52,5 \%)$ mini- porcos observou-se hiperatividade mucípara com catarro; em 17 (42,5\%), aumento da atividade mucípara; em dez (25\%), erosão. Os achados revelaram alta prevalência de lesões pré-ulcerativas nos estômagos dos miniporcos. A presença destas relacionou-se à gravidade macroscópica: quanto mais graves, mais intensa a paraqueratose.

Palavras-chave: miniporco, suíno, úlcera gástrica.

\begin{abstract}
Gastro-esophageal ulcers are one of the principal causes of reproduction pigs sudden death. This work aimed to evaluate the pathological findings of subclinical gastric lesions naturally occurring in mini pigs. Forty mini pigs with a mean weight of $39.0 \mathrm{~kg}$ and 17 months of age kept in animal facility, under constant temperature and humidity, and fed twice a day, were euthanized and necropsied. Their stomachs were collected and evaluated. Macroscopic lesions in aglandular portion were observed in 29 animals, and $37.5 \%$ of them were classified as grade $1,22.5 \%$ as grade 2 , and $12.5 \%$ as grade 3. In $29(72.5 \%)$ animals parakeratosis was microscopically seen: $35 \%$ had the moderate manner, $25 \%$ discreet and $12.5 \%$ severe one. Glandular lesions were more extensive in the cardia region, followed by antral and fundic. Thirty-six (90\%) animals had lesions in at least one of three regions. In $21(52.5 \%)$ mini pigs, it was observed mucipara hyperactivity with phlegm; in 17 (42.5\%), increased mucipara activity; and in ten $(25 \%)$, erosion. Findings revealed high prevalence of pre-ulcerative lesions in the stomachs of mini pigs. The presence of these was related to the macroscopic gravity: the more serious, more intense was the parakeratosis.
\end{abstract}

Keywords: gastric ulcer, minipigs, swine.

\section{Introdução}

O impacto da suinocultura sobre a economia mundial, especialmente no Brasil, torna indispensável o controle rigoroso das afecções que comprometem o rendimento econômico (Porto et al., 2004). Entre estas, destaca-se a úlcera gastroesofágica (UGE), um problema de ocorrência mundial que preocupa suinocultores, veterinários e a indústria de ração (Liesner et al., 2009). A UGE é uma doença multifatorial, figurando como uma das principais causas de morte súbita de suínos adultos em fase de reprodução, sendo mais frequente em animais criados intensivamente.

A similaridade morfofuncional com o homem elegeu a espécie suína ideal para a experimentação comparativa (Smith e Swindle, 2006), e está sendo, cada vez mais, utilizada como alternativa ao uso de cães e primatas em testes toxicológicos pré-clínicos de fármacos (Swandle et al., 2012). Foram desenvolvidas colônias de miniporcos com dimensões compatíveis para a utilização em laboratório, facilitando o seu emprego como modelo experimental em diversas áreas de pesquisa médicobiológica (Mariano, 2003). Notadamente, as vantagens de sua utilização são a facilidade de manuseio, redução de espaço e a quantidade de alimento.

O objetivo deste trabalho foi avaliar os achados anatomopatológicos das lesões gástricas subclínicas, notadamente as pré-ulcerativas, de ocorrência natural em miniporcos.

\section{Material e métodos}

Quarenta leitões (CEUA 127/2012) com peso médio de 39,0 kg e idade de 17 meses, mantidos no biotério do XX, em condições de temperatura e umidade constantes, alimentados duas vezes ao dia, foram eutanasiados e necropsiados. Os estômagos foram colhidos, cortados ao longo da curvatura maior, lavados e fotografados.

\footnotetext{
*Recebido em 1 de fevereiro de 2015 e aceito em 18 de novembro de 2015.

${ }^{* *}$ Professor, Universidade Federal Fluminense - UFF

***Médica Veterinária, NAL, Universidade Federal Fluminense - UFF

****Professor, Universidade Estadual do Norte Fluminense Darcy Ribeiro - UENF

Autor para correspondência: renatosilveira@vm.uff.br
} 
As lesões encontradas foram graduadas, segundo Silveira et al. (2014), de 0 a 5, sendo o grau 0 atribuído ao aspecto normal (liso, brilhoso e esbranquiçado); o grau 1, àquele com aparência parcialmente rugosa e com alguma área com pigmentação amarelada; o grau 2, aos com a região totalmente rugosa e espessada e com áreas de pigmentação amarelada; o grau 3 , aos com a região aglandular totalmente rugosa, espessada e com pigmentação amarelada em toda a sua superfície; o grau 4 , aos com a região totalmente rugosa, espessada, com pigmentação amarelada em toda a sua superfície e, ainda, mostrando áreas de erosão; e o grau 5 , àqueles com aparência totalmente rugosa, espessada, com pigmentação amarelada em toda a sua superfície e com áreas de ulceração.

Amostras das regiões aglandular (quadrilátero esofágico) e glandular (cárdica, fúndica e pilórica) foram colhidas e colocadas em frascos identificados contendo solução de formalina neutra tamponada a $10 \%$ e encaminhadas ao Laboratório de Morfologia e Patologia Animal, onde foram processadas e analisadas. $O$ processamento histotécnico seguiu a rotina para inclusão em parafina e os cortes foram corados por Hematoxilina e Eosina. Foram calculadas as frequências relativas das características aglandular e glandular, macro e microscópicas.

\section{Resultados e discussão}

A idade dos animais avaliados foi de 17 meses, portanto, maior que a idade de animais comerciais. Nestes, as úlceras gástricas podem afetar suínos de várias idades, mas as maiores taxas de ocorrência acontecem no intervalo 3-6 meses (Talummuk et al., 2010).

As alterações na região aglandular são de caráter multifatorial. Neste trabalho, apesar do esforço na tentativa de diminuí-las, garantindo condições adequadas na criação, foram observadas lesões notadamente pré-ulcerativas, ou seja, iniciais. Muitos fatores têm sido considerados como possíveis causas (Roosendaal et al., 2000) e tem, como principais responsáveis, os aspectos fisiológicos da espécie, componentes nutricionais e estruturais da dieta, microbiota residente e inúmeros fatores de manejo que contribuem de alguma forma para uma condição de estresse social dos animais, tais como o tipo e frequência do arraçoamento, superlotação, enfermidades, contaminação ambiental com poeira ou amônia, entre outros (Amory et al., 2006). Um destes fatores seria o fato de o estômago do suíno, ao contrário do de outros animais, ter pouca habilidade de mistura, com características fisiológicas para raramente esvaziar. $\mathrm{O}$ uso de rações concentradas, como utilizadas nesta pesquisa, leva a uma maior fluidificação e mistura do bolo alimentar, ocorrendo uma perda do gradiente de $\mathrm{pH}$ entre as regiões proximal e distal do estômago. Por este motivo, há uma exposição da região aglandular ao $\mathrm{pH}$ ácido e às enzimas. $\mathrm{O}$ aumento do $\mathrm{pH}$ na região fúndica estimula secreção ácida (Robertson e Hampson, 2001), agravando o quadro, pois a região aglandular não secreta o muco impermeabilizante (Knezevic et al., 2007). Estudos com o manejo alimentar e a granulometria dos produtos utilizados nas rações de miniporcos devem ser feitos para esclarecimento deste ponto.
Macroscopicamente, 29 animais apresentaram lesões na região aglandular (72,5\%), enquanto $11(27,5 \%)$ não revelaram alteração (Tabela 1). Vários autores relatam porcentagens acima de $70 \%$ de algum tipo lesão em seus estudos (Melnichouk, 2002; Yamasaki et al., 2006; Proietti et al., 2010; Swaby e Gregory, 2012), enquanto outros relatam abaixo desses percentuais (Dalla Costa et al., 2006).

Tabela 1: Graus de lesão na mucosa gástrica aglandular e glandular de miniporcos

\begin{tabular}{cccccccc}
\hline & Grau 0 & Grau 1 & Grau 2 & Grau 3 & & Total \\
\hline $\begin{array}{c}\text { Região } \\
\text { Aglandular }\end{array}$ & 11 & 15 & 9 & 5 & & & 40 \\
\hline Região & Normal & $\begin{array}{c}\text { Atividade } \\
\text { Mucípara }\end{array}$ & Erosão & $\begin{array}{c}\text { Hiperemia } \\
\text { Regional }\end{array}$ & $\begin{array}{c}\text { Exsudato } \\
\text { Neutrofilico }\end{array}$ & Catarro & \\
\hline $\begin{array}{c}\text { Glandular } \\
\text { (Antro) }\end{array}$ & 26 & 7 & 0 & 0 & 0 & 7 & 40 \\
\hline $\begin{array}{c}\text { Região } \\
\text { Glandular } \\
\text { (cárdica) }\end{array}$ & 9 & 8 & 6 & 1 & 5 & 11 & 40 \\
\hline $\begin{array}{c}\text { Região } \\
\text { Glandular } \\
\text { (fúndica) }\end{array}$ & 29 & 2 & 4 & 1 & 1 & 3 & 40 \\
\hline
\end{tabular}

Dos animais com lesão macroscópica, 15 foram classificados como grau $1(37,5 \%)$; nove, como grau $2(22,5 \%)$; e cinco, como grau 3 (12,5\%) (Tabela 1). O escore foi firmado em razão da ocorrência de lesões, na região aglandular, não se apresentarem de forma severa, privilegiando as lesões pré-iniciais ou iniciais não ulcerativas. De forma diferente, outros autores (Robertson et al., 2002; Dalla Costa et al., 2006; Yamasaki et al., 2006; Kopinski e McKenzie, 2007; Corrêa et al., 2008; Swaby e Gregory, 2012) utilizaram quatro graus em seus escores, visto que suas pesquisas foram realizadas com animais comerciais e não com os de biotérios. Nestes casos, a incidência de lesões mais graves é maior.

Em um matadouro inglês, Swaby e Gregory (2012), relataram que, de 9827 estômagos de suínos comerciais, cerca de $80 \%$ apresentaram algum tipo de lesão e, comparativamente a anos anteriores, a porcentagem de lesões graves diminuiu. Situação semelhante foi registrada por Oliveira et al. (2010) que, de 89,7\% de algum tipo de lesão na região aglandular, $83,1 \%$ foram iniciais. Uma explicação para esta situação era de que os animais com lesões mais graves, e com sinais de úlcera, não são enviados ao abate. Interesses econômicos e de bem-estar justificam o monitoramento do problema para determinação da prevalência e severidade das lesões gástricas (Friendship, 2004).

Apesar de se encontrarem com frequência lesões gástricas, no Brasil poucos são os relatos sobre análise histológica das mesmas (Yamasaki et al., 2009) e, quando encontrados, acontecem em animais de matadouro e não os de biotério, como no caso deste trabalho.

Microscopicamente, 29 animais $(72,5 \%)$ apresentaram paraqueratose. Destes, dez (25\%) tinham a forma discreta, $14(35 \%)$ a moderada e cinco $(12,5 \%)$ a acentuada (Gráfico 1). Estes resultados são semelhantes à prevalência de $75 \%$ encontrada por Marruchella et al. (2004) e 77,6\% por Yamasaki 


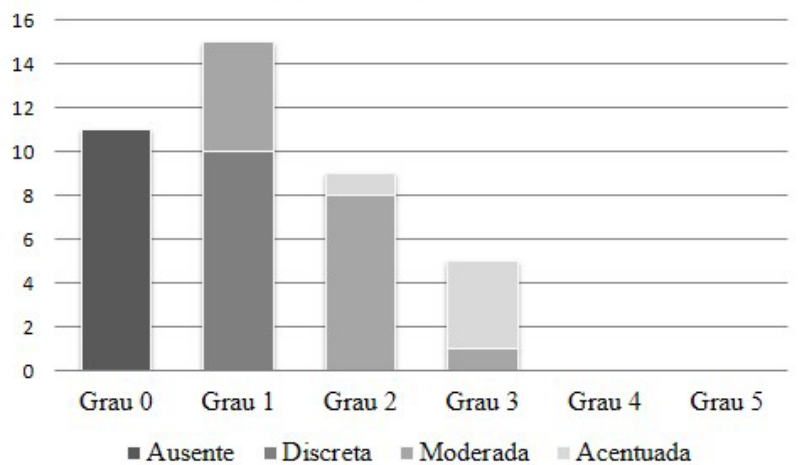

Gráfico 1: Graus de lesão da região aglandular de estômagos de miniporcos e intensidades de paraqueratose et al. (2009). Neste caso, os dados de animais comerciais se assemelham aos encontrados nos de biotério.

A paraqueratose é a resposta a um processo irritativo da mucosa, formada de epitélio pavimentoso estratificado corneificado, representado pela hiperplasia dos queratinócitos, quando aqueles superficiais se apresentam com núcleos picnóticos (necrosados) nas, camadas córneas que se descamam (Figura 1), ou seja, paraqueratóticas. Comparando porcas criadas intensivamente e extensivamente, Davies et al. (2001) observaram que as lesões gástricas consistiram, principalmente, em hiperqueratinização (paraqueratose) e coloração biliar, sendo mais extensas nas criadas intensivamente.

Comparando-se as lesões macro e microscópicas desta região, verificou-se que, dos 11 animais com grau 0 (27,5\%),

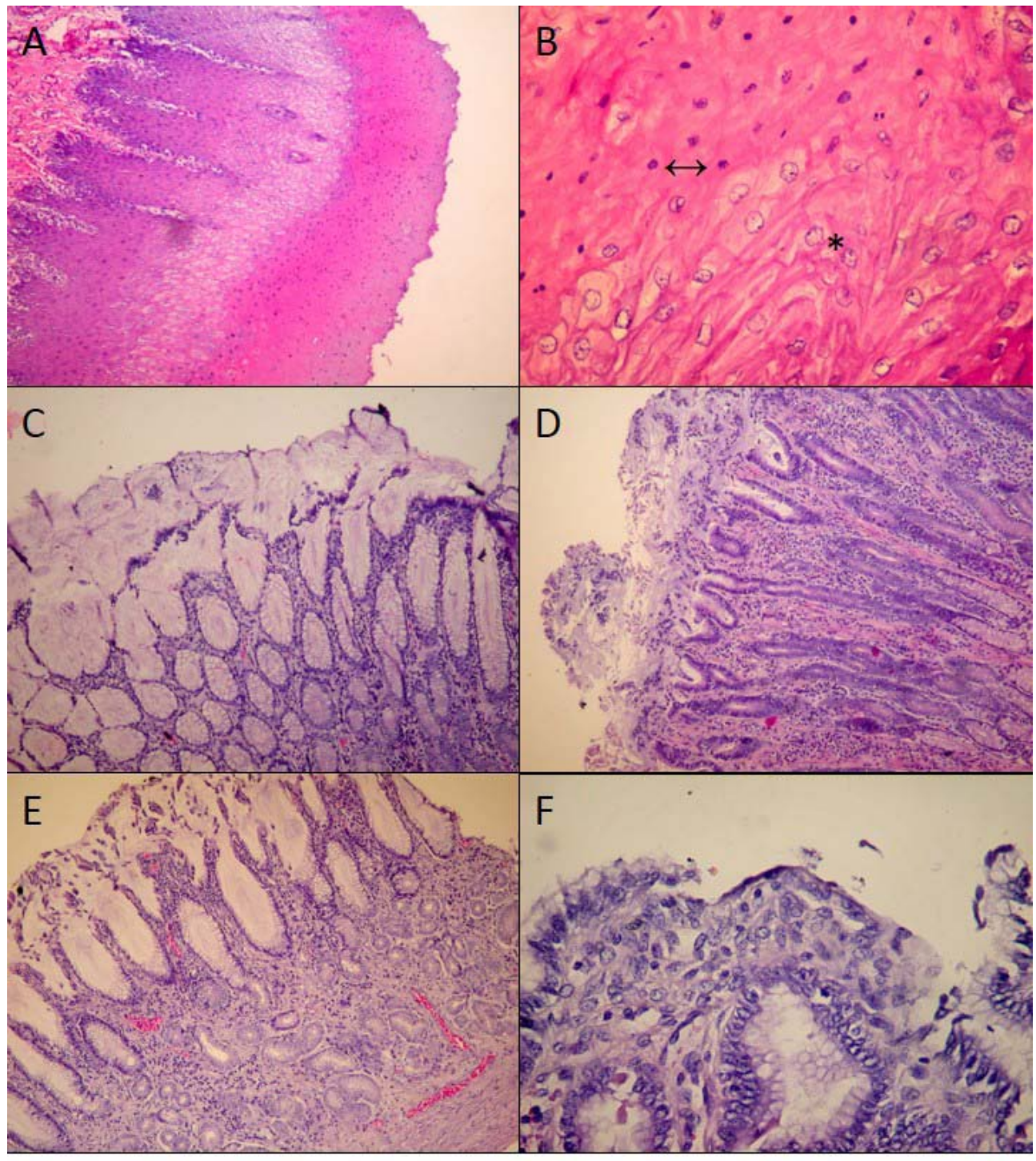

Figura 1: Fotomicrografia de estômagos de miniporco. H.E. A/B. Região aglandular com paraqueratose (seta/ núcleos picnóticos e asterisco/núcleos viáveis) (Objs. 10x e 40x, respectivamente). C. Região de glândulas cárdicas com atividade mucípara (Obj. 10x). D. Região de glândulas pilóricas, atividade mucípara com catarro (Obj. 10x). E. Região de glândulas fúndicas, hiperemia incipiente e regional (Obj. 10x). F. Região de glândulas cárdicas com erosão (Obj. 40x). 
nenhum apresentou paraqueratose. Dos 15 com grau 1, dez (25\%) apresentaram paraqueratose discreta e cinco $(12,5 \%)$ moderada. Dos nove com grau 2, oito (20\%) apresentaram paraqueratose moderada e um (2,5\%) acentuada. Dos cinco com grau 3, quatro (10\%) apresentaram paraqueratose acentuada e um $(2,5 \%)$ moderada (Gráfico 1$)$.

Do total de animais, 36 (90\%) apresentaram alteração em pelo menos uma das três regiões glandulares. Em 21 (52,5\%), houve hiperatividade mucípara com formação de catarro (muco misturado a debris etc). Em 17 deles (42,5\%), houve apenas aumento da atividade mucípara. Em dez (25\%) deles, houve erosão (perda das camadas superficiais,). Em seis (15\%), houve infiltrado neutrofílico e, em dois (5\%), hiperemia incipiente e regional (Figura 1).

As lesões glandulares foram mais extensas na região cárdica, seguida da antral e fúndica, mesmo resultado encontrado por Silva et al. (2001).

$\mathrm{Na}$ região das glândulas cárdicas, $31(77,5 \%)$ animais apresentaram alterações. Destes, 11 (27,5\%) apresentaram

\section{Referências}

AMORY, J.R.; MACKENZIE, A.M.; PEARCE, G.P. Factors in the housing environment of finisher pigs associated with the development of gastric ulcers. Veterinary Record, v. 158, n. 8, p. 260-264, 2006.

CORRÊA, A.M.R.C.; ZLOTOWSK, P.; BARCELLOS, D.E.S.N.; CRUZ, C.E.F.; DRIEMEIER, D. Gastric ulcers in pigs affected with postweaning multisystemic wasting syndrome. Pesquisa Veterinária Brasileira, v. 28, n. 12, p. 601-605, 2008.

DALLA COSTA, O.A.; COLDEBELLA, A.; COSTA, M.J.R.P.; FAUCITANO, L.; PELOSO, J.V.; LUDKE, J.V.; SCHEUERMANN G.N. Período de descanso dos suínos no frigorífico e seu impacto na perda de peso corporal e características do estômago. Ciência Rural, v. 36, n. 5, p. 1582-1588, 2006.

DAVIES, Z.E.; GUISE, H.J.; PENNY, R.H.; SIBLY, R.M. Effects of stone chewing by outdoors sows on their teeth and stomachs. Veterinary Record, v.149, n.1, p.9-11, 2001.

FRIENDSHIP, R.M. Gastric ulceration in swine. Journal of Swine Health and Production, v.12, n. 1, p. 34-35, 2004.

KNEZEVIC, M.; KOVACEVIC, S.A.; ALEKSIC, Z. Cell proliferation in pathogenesis of esophagogastric lesions in pigs. International Review of Citology, v. 260, p.1-34, 2007.

KOPINSKI, J.S. e McKENZIE, R.A. Oesophagogastric ulceration in pigs: a visual morphological scoring guide. Australian Veterinary Journal, v. 85, n. 9, p. 356-361, 2007.

LIESNER, V.G.; TAUBE, V.; MAREK, S.L.; BEINEKE, A.; KAMPHUES, J. Integrity of gastric mucosa in reared piglets: effects of physical form of diets (meal/pellets), preprocessing grinding (coarse/fine) and addition of lignocelluloses (0/2.5\%). Journal of Animal Physiology and Animal Nutrition, v. 93, n. 3, p. 373-380, 2009.

MARIANO, M. Minisuíno (minipig) na pesquisa biomédica experimental. O Minipig BR 1. Acta Cirúrgica Brasileira, v.18, n. 5, p. 387-391, 2003.

MARRUCELLA, G.; DI LEONARDO, M.; DI GUARDO, G.; ROMANUCCI, M.; MARA, M.; TISCAR, P.G.; MOSCA, F.; DELLA SALDA, L. Heat shock proteins (HSPs) 27, 72 and 73 in normal and pre-ulcerative mucosa of the gastric pars oesophagea in swine. Journal of Comparative Pathology, v.131, n.1, p. 10-17, 2004. hiperatividade mucípara com formação de catarro, oito $(20 \%)$ apenas aumento da atividade mucípara, seis (15\%) erosão, cinco $(12,5 \%)$ exsudato neutrofílico e um $(2,5 \%)$ hiperemia (Tabela 1$)$.

$\mathrm{Na}$ região do antro (glândulas pilóricas), 14 (35\%) animais apresentaram alterações. Destes, sete $(17,5 \%)$ apresentaram atividade mucípara e sete $(17,5 \%)$ atividade mucípara com formação de catarro (Tabela 1).

$\mathrm{Na}$ região das glândulas fúndicas, $11(27,5 \%)$ suínos apresentaram alterações. Destes, quatro (10\%) apresentaram erosão, três $(7,5 \%)$ atividade mucípara com catarro, dois $(5 \%)$ atividade mucípara, um (2,5\%) apresentou exsudato neutrofílico e um $(2,5 \%)$ hiperemia (Tabela 1$)$.

\section{Conclusões}

Os achados revelaram alta prevalência de lesões pré-ulcerativas nos estômagos dos miniporcos. A presença destas relacionou-se à gravidade macroscópica: quanto mais graves, mais intensa a paraqueratose.

MELNICHOUK, S.I. Mortality associated with gastric ulceration in swine. Canadian Veterinary Journal, v. 43, n. 3, p. 223-225, 2002.

OLIVEIRA, S.J.; BERNARDI, R.T.; VOGT, F.I.; SCARTEZZINI, M.; HEPP, D.; LUNG, V.R. Úlceras gástricas em suínos de abate: cultivo de Arcobacter spp. a partir de estômagos com diferentes graus de lesões. Acta Scientiae Veterinariae, v. 38, n. 4, p. 351356, 2010.

PORTO, R.N.G.; SOBESTIANSKY, J.; MATOS, M.P.C.; MEIRINHOS, M.L.G. Aspectos histopatológicos do sistema urinário de matrizes suínas descartadas. Ciência Animal Brasileira, v. 5, n. 2, p. 109-112, 2004.

PROIETTI, P.C.; BIETTA, A.; LEPRI, E.; DAVIDSON, I.; FRANCIOSINI, M.P. Detection of Helicobacter spp. in gastric, fecal and saliva samples from swine affected by gastric ulceration. Journal of Veterinary Sciense, v.11, n. 3, p. 221-225, 2010.

ROBERTSON, I.D.; ACCIOLY, J.M.; MOORE, K.M.; DRIESEN, S.J.; PETHICK, D.W.; HAMPSON, D.J. Risk factors for gastric ulcers in Australian pigs at slaughter. Preventive Veterinary Medicine, v. 53, n. 4, p. 293-303, 2002.

ROBERTSON, I.D. e HAMPSON, D.J. Investigating oesophagogastric ulcers in Australia. Pig Progress, v. 17, n. 6, p. 14-16, 2001.

ROOSENDAAL, R.; VOS, J.H.; ROUMEN, T.; VAN VUGT, R.; CATTOLI, G.; BART, A.; KLAASEN, H.L.B.M.; KUIPERS, E.J.; VANDENBROUCKE-GRAULS, C.M.J.E.; KUSTERS, J.G. Slaughter pigs are commonly infected by closely related but distinct gastric ulcerative lesion-inducing Gastrospirilla. Journal of Clinical Microbiology, v. 38, n. 7, p. 2661-2664, 2000.

SILVA, J.C.P.; SANTOS, J.L.; BARBOSA, A.J.A. Gastrite em suínos: frequência, relação com a úlcera gástrica e com a densidade de células endócrinas do estômago. Revista Brasileira de Ciências Veterinárias, v. 8, n. 1, p. 40-43, 2001.

SILVEIRA, R.L.; CRUZ, A.C.M.; DEGANI, V.A.N.; WEBER, F.A.G.C.; CARVALHO, E.C.Q. Helicobacteriose em leitões: imuno-histoquímica em amostras colhidas por meio de gastroscopia. Arquivo Brasileiro de Medicina Veterinária e Zootecnia, v. 66, n. 6, p. 1681-1686, 2014.

SMITH, A.C. e SWINDLE, M.M. Preparation of swine for the laboratory. Journal of Institute for Laboratory Animal Research, v.47, n.4, p.358-363, 2006. 
SWABY, H. e GREGORY, N.G. A note on frequency of gastric ulcers detected during post mortem examination at a pig abattoir. Meat Science, v. 90, n.1, p. 269-271, 2012.

SWINDLE, M.M.; MAKIN, A.; HERRON, A.J.; CLUBB JR, F.J.; FRAZIER, K.S. Swine as model in Biomedical Research and Toxicology Testing. Veterinary Pathology, v. 49, n. 2, p. 344-356, 2012.

TALUMMUK, S.; KUNALINTIP, R.; MAKHANON, M. Case report of gastric ulcers in grower pigs affected with persistent Porcine Respiratory disease Complex (PRDC). Proceedings of $21^{\text {th }}$ IPVS Congress, Vancouver, Canada, p.745, 2010.
YAMASAKI, L.; ASSIS, M.S.; ROSSETO, V.J.V.; BRACARENSE, A.N.F.R.L. Lesões gástricas em suínos: Ocorrência e relação com o gênero, peso ao abate e presença de Helicobacter spp. Ciências Agrárias, v. 27, n. 3, p. 463-470, 2006.

YAMASAKI, L.; BOSELLI-GROTTI, C.C.; ALFIERI, A.A.; SILVA, E.O.; OLIVEIRA, R.L.; CAMARGO, P.L.; BRACARENSE, A.P.F.R.L. Alterações histológicas da pars esophagea de suínos e sua relação com Helicobacter spp. Arquivo Brasileiro de Medicina Veterinária e Zootecnia, v. 61, n. 3, p. 553-560, 2009. 